\title{
Feasibility and efficacy of enhanced recovery after surgery protocol in Chinese elderly patients with intracranial aneurysm
}

This article was published in the following Dove Medical Press journal:

Clinical Interventions in Aging

\author{
Hui Han* \\ Songtao Guo* \\ Hao Jiang \\ $\mathrm{XiWu}$
}

Department of Neurosurgery, Inner Mongolia Xing'an Meng People's Hospital, Ulanhot 137400, China

*These authors contributed equally to this work
Correspondence: Xi Wu

Department of Neurosurgery, Inner Mongolia Xing'an Meng People's Hospital, Ulanhot 137400, China

Email1617582659@qq.com
Objective: Intracranial aneurysm is a kind of severe intracranial disease mainly responsible for subarachnoid hemorrhage, and the rupture of intracranial aneurysm results in a mortality rate of $30 \%-40 \%$. For the first time in the world, this study aimed to assess the feasibility and efficacy of enhanced recovery after surgery (ERAS) protocol in Chinese elderly patients with intracranial aneurysm.

Methods: In this study, 300 elderly patients with intracranial aneurysm were recruited and divided into two groups as follows: ERAS group $(n=150$, ERAS protocol) and control group ( $\mathrm{n}=150$, conventional management).

Results: Age of whole cohort was 65 (64-67) years with 140 males (46.7). There was no difference between two groups in baseline features of patients, such as age, sex, medical histories, percentages of aneurysmal location, aneurysmal number $>1$ per patient, aneurysmal diameter $>5 \mathrm{~mm}$, or lobular aneurysm ( $P>0.05$ for all). There was no occurrence of death in two groups. Compared with those in the control group, patients in the ERAS group had significantly shorter length of hospital stay $(P<0.05)$. Between two groups, patients had not only similar Glasgow Outcome Scale (GOS) and Modified Rankin Scale (MRS) at discharge but also occurrence of readmission at follow-up ( $P>0.05$ for all). Patients in the ERAS group had significantly higher GOS and lower MRS at follow-up $(P<0.05$ for all).

Conclusion: ERAS protocol significantly shortened the length of hospital stay and improved GOS and MRS without any increase in the mortality or readmission in Chinese elderly patients with intracranial aneurysm.

Keywords: elderly, enhanced recovery after surgery, intracranial aneurysm

\section{Introduction}

Intracranial aneurysm is a kind of severe intracranial disease mainly responsible for subarachnoid hemorrhage, and the rupture of intracranial aneurysm results in a mortality rate of $30 \%-40 \% .^{1,2}$ Although significant advance has been made in surgical operation of intracranial aneurysm, there is still poor post-operative recovery in patients with intracranial aneurysm. ${ }^{1,3}$ In addition to those related to disease itself and surgical operation, stress response, persistent pain, and metabolic demand serve as critical pathogenic factors in post-operative recovery. ${ }^{4}$ Enhanced recovery after surgery (ERAS) offers a series of approaches, such as professional education, nutritional care, improved anesthesia, and fluid management. ${ }^{5}$ ERAS protocol has been applied for various operations, such as colonic, pancreaticoduodenectomy, cystectomy, gastrectomy, and rectal/pelvic operation. ${ }^{6-10}$ ERAS protocol has the chance to not only accelerate the recovery of different patients but also shorten the length of 
hospital stay. ${ }^{11}$ However, there is nearly no study to analyze the feasibility and efficacy of ERAS protocol in Chinese elderly patients with intracranial aneurysm. For the first time in the world, this study aimed to assess the feasibility and efficacy of ERAS protocol in Chinese elderly patients with intracranial aneurysm.

\section{Methods}

\section{Study patients}

From January 2011 to July 2017, 300 patients at the Department of Neurosurgery, Inner Mongolia Xing'an Meng People's Hospital were recruited in this study. The inclusion criteria include the following: 1) age over 60 years; 2) medical history, clinical appearance (dizziness, headache, and nerve localization signs), and diagnostic imaging (computed tomography angiography [CTA] or digital subtraction angiography [DSA]) supported the diagnosis of intracranial aneurysm; 3) surgical treatment of intracranial aneurysm; and 4) the ability to understand and participate in this study. The exclusion criteria include the following: 1) contraindications to surgical treatment of intracranial aneurysm and 2) severe cardiac, hepatic, renal, and other systematic diseases. The study protocol has been approved by the ethics committee of Inner Mongolia Xing'an Meng People's Hospital, and it conforms to the Declaration of Helsinki. Each participant provided written informed consent to be included in the study.

\section{Study protocol}

Patients were randomly allocated to two groups as follows: ERAS group ( $n=150$, ERAS protocol) and control group ( $n=150$, conventional management). Surgical treatment of intracranial aneurysm was performed by united team with full experience. Patients were placed in the supine position, and operation was performed under general anesthesia. Minimally invasive surgical approach through small pterion or inferolateral forehead was applied in all patients. Intracranial aneurysm was treated with clipping. Operation was monitored by electroencephalogram and somatosensory evoked potential. Blood flow and its patency were assessed during the operation by microvascular Doppler ultrasound. Fluorescence angiography was applied to assess the patency of parental artery and perforating artery.

\section{ERAS protocol}

ERAS protocol was performed as shown in Table $1 .{ }^{12}$ In addition to educating the patient on surgical risks and benefits as

Table I ERAS strategy performed in this study

\begin{tabular}{|c|c|c|}
\hline Strategy & Control group & ERAS group \\
\hline \multicolumn{3}{|l|}{ Preoperative } \\
\hline Preoperative education & Conventional & Detailed (ERAS strategy) \\
\hline Oral diet & Nothing for 8 hours before operation & Fluids until 2 hours and solids until 6 hours before operation \\
\hline Short-acting anxiolytics & Occasionally & Conventional \\
\hline Corticosteroid and antiemetic & Occasionally & Conventional \\
\hline \multicolumn{3}{|l|}{ Operative } \\
\hline Patient-controlled analgesia pump & No & Yes \\
\hline Nasogastric tube & Conventional & Minimized \\
\hline Room temperature & Conventional & $>25^{\circ} \mathrm{C}$ \\
\hline Heated liquid & No & Yes \\
\hline Warm air & No & Yes \\
\hline Fluid administration & Conventional & Goal-directed manner \\
\hline \multicolumn{3}{|l|}{ Postoperative } \\
\hline Urinary catheter removal & Three days after operation & One day after operation \\
\hline Water & Twelve hours after operation & Six hours after operation \\
\hline Liquid food & Two days after operation & One day after operation \\
\hline Semiliquid diet & Three or four days after operation & Two days after operation \\
\hline Normal diet & Five or six days after operation & Three days after operation \\
\hline Fluid administration & Conventional & Restricted $(<2,500 \mathrm{~mL})$ \\
\hline Patient-controlled analgesia pump & No & Yes \\
\hline Pulmonary physical therapy & No & Conventional \\
\hline Instrument after discharge & Self-determination of patients & Phone reminder, detailed and frequent \\
\hline
\end{tabular}

Abbreviation: ERAS, enhanced recovery after surgery. 
part of the control protocol, pre-operative education of the ERAS protocol included the information about appointments and instructions, testing and drugs, eating and drinking, smoking and alcohol, expectations about disposition, and surgical site care. ${ }^{4}$ In addition to post-operative instrument of the control protocol at clinics after discharge based on the self-determination of patients, patients received phone reminder from surgical doctors within 48 hours of discharge and every week after operation to assess pain management, identify patient concerns, and clarify follow-up instrument.

\section{Outcome measure}

All patients were followed up for 1 year, and there were no patients lost during the follow-up. The primary outcomes were assessed by the mortality and the length of hospital stay after operation (the number of days from operation to discharge) during the first year after operation. The secondary outcome was assessed by the readmission during the first year after operation. Glasgow Outcome Scale (GOS) and Modified Rankin Scale (MRS) were applied to assess neurological function and prognostic performance at discharge and the first year after operation.

\section{Statistical analyses}

Continuous variables with normal distribution were described using mean and SD and compared between two groups using Student's $t$-test. Continuous variables with skewed distribution were described using median and IQR and compared between two groups using the Mann-Whitney $U$ test. Categorical variables were described with number and percentage and compared between two groups using the chi-squared test. Statistical analysis was performed using
SPSS 18.0 software (SPSS Inc., Chicago, IL, USA). $P<0.05$ was considered to be statistically significant.

\section{Results}

Age of whole cohort was 65 (64-67) years with 140 males (46.7\%). As shown in Table 2, there was no difference between two groups in baseline features of patients, such as age, sex, medical histories, percentages of aneurysmal location, aneurysmal number $>1$ per patient, aneurysmal diameter $>5 \mathrm{~mm}$, or lobular aneurysm $(P>0.05$ for all). Clinical outcomes of patients were compared between two groups in Table 3. There was no occurrence of death in two groups. Compared with those in the control group, patients in the ERAS group had significantly shorter length of hospital stay $(P<0.05)$. Between two groups, patients had not only similar GOS and MRS at discharge but also occurrence of readmission at follow-up ( $P>0.05$ for all). Patients in the ERAS group had significantly higher GOS and lower MRS at follow-up $(P<0.05$ for all).

\section{Discussion}

Intracranial aneurysm is a kind of severe intracranial disease mainly responsible for subarachnoid hemorrhage, and the rupture of intracranial aneurysm results in a mortality rate of $30 \%-40 \% .^{1,2}$ Although significant advance has been made in surgical operation of intracranial aneurysm, there is still poor post-operative recovery in patients with intracranial aneurysm. ${ }^{1,3}$ Surgical treatment of intracranial aneurysm often requires complex operation, as well as careful preoperative preparation, intraoperative care, and post-operative management. ${ }^{12}$ Enhanced recovery is significant in patients with intracranial aneurysm but evidence-based protocol on

Table 2 Baseline features of all patients divided into ERAS and control groups

\begin{tabular}{|c|c|c|c|c|}
\hline Features & $\begin{array}{l}\text { All patients } \\
(n=300)\end{array}$ & $\begin{array}{l}\text { Control group } \\
(n=150)\end{array}$ & $\begin{array}{l}\text { ERAS group } \\
(n=150)\end{array}$ & $P$-value \\
\hline Age, years & $65(64-67)$ & $65(64-67)$ & $65(64-68)$ & 0.451 \\
\hline Males, n (\%) & $140(46.7)$ & $69(46.0)$ & $71(47.3)$ & 0.817 \\
\hline Hypertension, n (\%) & I 58 (52.7) & $77(51.3)$ & $81(54.0)$ & 0.644 \\
\hline Diabetes mellitus, $\mathrm{n}(\%)$ & $85(28.3)$ & $39(26.0)$ & $46(30.7)$ & 0.370 \\
\hline Aneurysmal location, $\mathrm{n}(\%)$ & & & & 0.705 \\
\hline Posterior communicating and anterior choroidal aneurysm & $151(50.3)$ & $79(52.7)$ & $72(48.0)$ & \\
\hline Middle cerebral aneurysm & $70(23.3)$ & $34(22.7)$ & $36(24.0)$ & \\
\hline Anterior communicating aneurysm & $79(26.3)$ & $37(24.7)$ & $42(28.0)$ & \\
\hline Aneurysmal number $>$ I per patient, $\mathrm{n}(\%)$ & $21(7.0)$ & $10(6.7)$ & II (7.3) & 0.821 \\
\hline Aneurysmal diameter $>5 \mathrm{~mm}, \mathrm{n}(\%)$ & $63(21.0)$ & $29(19.3)$ & $34(22.7)$ & 0.478 \\
\hline Lobular aneurysm, n (\%) & $84(28.0)$ & $38(25.3)$ & $46(30.7)$ & 0.304 \\
\hline
\end{tabular}

Abbreviation: ERAS, enhanced recovery after surgery. 
Table 3 Clinical outcomes of all patients divided into ERAS and control groups

\begin{tabular}{|c|c|c|c|}
\hline Outcomes & Control group $(n=150)$ & ERAS group $(n=\mid 50)$ & $P$-value \\
\hline Length of hospital stay, days & $10(8-12)$ & $9(7-I I)$ & 0.019 \\
\hline Readmission, n (\%) & $4(2.7)$ & $2(1.3)$ & 0.680 \\
\hline \multicolumn{4}{|l|}{ GOS at discharge, n (\%) } \\
\hline 5 & $143(95.3)$ & $147(98.0)$ & 0.198 \\
\hline 4 & $7(4.7)$ & $3(2.0)$ & \\
\hline \multicolumn{4}{|l|}{ MRS at discharge, $\mathbf{n}(\%)$} \\
\hline $0-1$ & $134(89.3)$ & $143(95.3)$ & 0.148 \\
\hline 2 & $9(6.0)$ & $4(2.7)$ & \\
\hline 3 & $7(4.7)$ & $3(2.0)$ & \\
\hline \multicolumn{4}{|l|}{ GOS at follow-up, n (\%) } \\
\hline 5 & $138(92.0)$ & $146(97.3)$ & 0.040 \\
\hline 4 & $12(8.0)$ & $4(2.7)$ & \\
\hline \multicolumn{4}{|l|}{ MRS at follow-up, $\mathbf{n}(\%)$} \\
\hline $0-1$ & $126(84.0)$ & $140(93.3)$ & 0.034 \\
\hline 2 & $12(8.0)$ & $6(4.0)$ & \\
\hline 3 & $12(8.0)$ & $4(2.7)$ & \\
\hline
\end{tabular}

Abbreviations: ERAS, enhanced recovery after surgery; GOS, Glasgow Outcome Scale; MRS, Modified Rankin Scale.

perioperative care for this population is lacking. For the first time in the world, this study confirmed that the ERAS protocol significantly shortened the length of hospital stay and improved GOS and MRS without any increase in the mortality or readmission in Chinese elderly patients with intracranial aneurysm.

Stress response, persistent pain, and metabolic demand related to surgical operation delay the recovery of patients after operation. ${ }^{4}$ ERAS protocol has been introduced as a way of optimizing perioperative care for different operations, such as colonic, pancreaticoduodenectomy, cystectomy, gastrectomy, and rectal/pelvic operation. ${ }^{6-10}$ Improving perioperative care in an evidence-based and structured manner is the primary goal of the ERAS protocol. Evaluation of the ERAS protocol in patients with these diseases has shown that these patients had shorter length of hospital stay and experienced significant improvements in recovery after operation. ${ }^{12}$ ERAS protocol has revolutionized the way of perioperative care in surgical field. However, no evidence has been offered to support the ERAS protocol in Chinese elderly patients with intracranial aneurysm. In this study, the ERAS protocol offers a series of approaches, such as professional education, nutritional care, improved anesthesia, and fluid management. ${ }^{5}$ It is no surprise for these approaches to alleviate stress response, persistent pain, and metabolic demand related to surgical operation and promote the recovery of patients with intracranial aneurysm after operation. ${ }^{12}$

\section{Conclusion}

For the first time in the world, this study demonstrated that the ERAS protocol significantly shortened the length of hospital stay and improved GOS and MRS without any increase in the mortality or readmission in Chinese elderly patients with intracranial aneurysm.

\section{Disclosure}

The authors report no conflicts of interest in this work.

\section{References}

1. Greving JP, Wermer MJ, Brown RD, et al. Development of the PHASES score for prediction of risk of rupture of intracranial aneurysms: a pooled analysis of six prospective cohort studies. Lancet Neurol.2014; 13(1):59-66.

2. Wiebers DO. Unruptured intracranial aneurysms: natural history, clinical outcome, and risks of surgical and endovascular treatment. The Lancet. 2003;362(9378):103-110.

3. Connolly ES Jr, Rabinstein AA, Carhuapoma JR, et al. Guidelines for the management of aneurysmal subarachnoid hemorrhage: a guideline for healthcare professionals from the American Heart Association/American Stroke Association. Stroke. 2012;43(6):1711-1737.

4. Ali ZS, Ma TS, Ozturk AK, et al. Pre-optimization of spinal surgery patients: Development of a neurosurgical enhanced recovery after surgery (ERAS) protocol. Clin Neurol Neurosurg. 2018;164:142-153.

5. Kehlet H. Multimodal approach to control postoperative pathophysiology and rehabilitation. Br J Anaesth. 1997;78(5):606-617.

6. Gustafsson UO, Scott MJ, Schwenk W, et al. Guidelines for perioperative care in elective colonic surgery: Enhanced Recovery After Surgery (ERAS(®)) Society recommendations. World J Surg. 2013;37(2): 259-284.

7. Lassen K, Coolsen MM, Slim K, et al. Guidelines for perioperative care for pancreaticoduodenectomy: Enhanced Recovery After Surgery (ERAS $®)$ Society recommendations. World J Surg. 2013;37(2):240-258. 
8. Cerantola Y, Valerio M, Persson B, et al. Guidelines for perioperative care after radical cystectomy for bladder cancer: Enhanced Recovery After Surgery (ERAS(®)) society recommendations. Clin Nutr. 2013;32(6): 879-887.

9. Mortensen K, Nilsson M, Slim K, et al. Consensus guidelines for enhanced recovery after gastrectomy: Enhanced Recovery After Surgery (ERAS®) Society recommendations. Br J Surg. 2014;101(10): 1209-1229.

10. Nygren J, Thacker J, Carli F, et al. Guidelines for perioperative care in elective rectal/pelvic surgery: Enhanced Recovery After Surgery (ERAS(®)) Society recommendations. World J Surg. 2013;37(2): 285-305.
11. Kim JW, Kim WS, Cheong JH, Hyung WJ, Choi SH, Noh SH. Safety and efficacy of fast-track surgery in laparoscopic distal gastrectomy for gastric cancer: a randomized clinical trial. World J Surg. 2012; 36(12):2879-2887.

12. Dort JC, Farwell DG, Findlay M, et al. Optimal Perioperative care in major head and neck cancer surgery with free flap reconstruction: a consensus review and recommendations from the enhanced recovery after surgery society. JAMA Otolaryngol Head Neck Surg. 2017;143(3): $292-303$.
Clinical Interventions in Aging

\section{Publish your work in this journal}

Clinical Interventions in Aging is an international, peer-reviewed journal focusing on evidence-based reports on the value or lack thereof of treatments intended to prevent or delay the onset of maladaptive correlates of aging in human beings. This journal is indexed on PubMed Central, MedLine,

\section{Dovepress}

CAS, Scopus and the Elsevier Bibliographic databases. The manuscript management system is completely online and includes a very quick and fair peer-review system, which is all easy to use. Visit http://www.dovepress. $\mathrm{com} /$ testimonials.php to read real quotes from published authors. 